\title{
BIODIVERSITY OF MEXICAN TERRESTRIAL ARTHROPODS (ARACHNIDA AND HEXAPODA): A BIOGEOGRAPHICAL PUZZLE
}

\author{
Juan J. MORRONE1 \& Juan MÁRQUEZ² \\ ${ }^{1}$ Museo de Zoología "Alfonso L. Herrera", Departamento de Biología Evolutiva, Facultad \\ de Ciencias, Universidad Nacional Autónoma de México (UNAM), Apdo. postal 70-399, \\ 04510 Mexico D.F., MÉXICO. \\ 2Laboratorio de Sistemática Animal, Centro de Investigaciones Biológicas, Universidad \\ Autónoma del Estado de Hidalgo (UAEH), Apdo. postal 1-69, Plaza Juárez, \\ Pachuca, 42001 Hidalgo, MÉXICO.
}

\begin{abstract}
RESUMEN
Presentamos una revisión general del conocimiento de los artrópodos terrestres de México y algunas hipótesis para permitir explicar su complejidad biogeográfica, traducidas en regiones biogeográficas, dominios, provincias y nodos panbiogeográficos. Se reconocen dos regiones biogeográficas (Neártica y Neotropical) y una zona de transición. Dentro de ellas, se reconocen cinco dominios (Neártico Californiano, Neártico Continental, Mexicano de Montaña, Antillano y Mesoamericano) y 14 provincias (California, Baja California, Sonora, Altiplano Mexicano, Tamaulipas, Sierra Madre Occidental, Sierra Madre Oriental, Faja Volcánica Transmexicana, Cuenca del Balsas, Sierra Madre del Sur, Península de Yucatán, Golfo de México, Costa del Pacífico Mexicano y Chiapas). Quince áreas geográficas donde se intersectan tres provincias biogeográficas diferentes se consideran nodos panbiogeográficos: California Norte, Sonora, Sonora-Chihuahua-Sinaloa, Jalisco-Nayarit, JaliscoZacatecas, Monterrey (Nuevo León), Ciudad Victoria (Tamaulipas), noroeste de Michoacán, norte de Guerrero, Hidalgo-Puebla, Orizaba-Córdoba (Veracruz), Huajuapan de León (Oaxaca), Sierra de Juárez (Oaxaca), sur de Oaxaca y El Ocote (Chiapas). La Zona de Transición Mexicana presenta la mayor mezcla biótica entre elementos neárticos y neotropicales. Las especies distribuidas en las tierras bajas cerca de las costas de México poseen mayormente afinidades neotropicales y se detectan más al norte de lo que ha sido hipotetizado previamente. Los nodos poseen una mayor biodiversidad, con afinidades biogeográficas diferentes. Algunos de ellos se encuentran bajo protección por su alta biodiversidad. Ninguno de ellos ha sido estudiado adecuadamente, $\mathrm{y}$ las investigaciones futuras serán muy importantes para seleccionar más sitios con alta biodiversidad y especies que representen linajes e historias biogeográficas diferentes.
\end{abstract}

Palabras clave: biogeografía, insectos, arácnidos, región Neotropical, región Neártica.

\begin{abstract}
We present a general review of the knowledge on Mexican terrestrial arthropods and a set of hypotheses to help explain their biogeographic complexity, translated into biogeographic regions, dominions, provinces, and panbiogeographic nodes. Two biogeographic regions (Nearctic and Neotropical) and one transition zone are recognized. Within them, five dominions (Californian Nearctic,
\end{abstract}


Continental Nearctic, Mexican Mountain, Antillean, and Mesoamerican) and 14 provinces (California, Baja California, Sonora, Mexican Plateau, Tamaulipas, Sierra Madre Occidental, Sierra Madre Oriental, Transmexican Volcanic Belt, Balsas Basin, Sierra Madre del Sur, Yucatán Peninsula, Mexican Gulf, Mexican Pacific Coast, and Chiapas) are recognized. Fifteen geographic areas where three different biogeographic provinces intersect are considered panbiogeographic nodes: California Norte, Sonora, Sonora-Chihuahua-Sinaloa, Jalisco-Nayarit, Jalisco-Zacatecas, Monterrey (Nuevo León), Ciudad Victoria (Tamaulipas), northwestern Michoacán, northern Guerrero, Hidalgo-Puebla, Orizaba-Córdoba (Veracruz), Huajuapan de León (Oaxaca), Sierra de Juárez (Oaxaca), southern Oaxaca, and El Ocote (Chiapas). The Mexican Transition Zone presents the highest level of biotic mixture between Nearctic and Neotropical elements. Species distributed in the lowlands near the Mexican coasts have mostly Neotropical affinities, and can be detected further north than previously hypothesized. Nodes possess a higher biodiversity, with different biogeographic affinities. Some of them are currently under protection for their high biodiversity. None of them has been studied adequately, and future research will be very important to select more sites with high biodiversity, and species representing different lineages and biogeographic histories.

Key words: biogeography, insects, arachnids, Neotropical region, Nearctic region.

\section{INTRODUCTION}

Mexican biodiversity is one of the highest in the world and endemicity for several taxa is extensive (Mittermeier 1988; Dirzo 1992). Arachnids and hexapods are especially well represented in the country, with about 38,000 known species and 8,800 endemic species (Llorente Bousquets et al. 1996a, 2000, 2004; Llorente Bousquets \& Morrone 2002). In addition to the physiographic, climatic, and ecological complexity of the country (Llorente Bousquets et al. 1996b), a frequent historical explanation for this high diversity and endemicity has been the particular placement of the country, between the Nearctic and Neotropical regions (Llorente Bousquets 1996; Morrone 2001a). In contrast to Simpson's (1980) "splendid isolation" to explain South American mammal diversity, this would be a "splendid mixture" of different biotic elements or a "biotic crossroads" (Liebherr 1994). In order to elaborate this explanation, Halffter (1962, 1965, 1972, 1974, 1976, 1978, 1987, 2003; see also Llorente Bousquets 1996 \& Reyes-Castillo 2003) developed the concept of the Mexican Transition Zone, as "a complex and varied area in which the Neotropical and the Nearctic faunas overlap" (Halffter 1987: 95). This area includes the southwestern United States, all of Mexico, and a large part of Central America extending to the Nicaraguan lowlands. Within the Mexican Transition Zone, Halffter categorized three distributional patterns: Nearctic, Paleoamerican, and Mesoamerican.

Halffter's theory can be tested with current biogeographic approaches. For example, Morrone \& Márquez (2001) undertook a track analysis of several beetle (Coleoptera) taxa from the Mexican Transition Zone, finding two generalized tracks: a northern generalized track, comprising basically mountain areas within the Sierra 
Madre Occidental, the Sierra Madre Oriental, the Transmexican Volcanic Belt, the Balsas Basin, and the Sierra Madre del Sur; and a southern generalized track, comprising basically lowland areas, in Chiapas, the Mexican Gulf, and the Mexican Pacific Coast, reaching in the south the Panamanian Isthmus. These generalized tracks agree basically with Halffter's ideas, although they are insufficient to account for the complex biogeographic patterns found in the country (Morrone 2005).

Our purpose is to present a general review of the knowledge on Mexican terrestrial arthropods and a set of hypotheses to help explain Mexican biogeographic complexity. These hypotheses concern generalized tracks and areas of endemism - herein translated into biogeographic regions, dominions, and provinces - that represent statements of primary biogeographic homology, which can be tested by future cladistic biogeographic analyses.

\section{MEXICAN TERRESTRIAL ARTHROPODS}

The Biologia Centrali-Americana, edited by Godman \& Salvin (1879-1915), constitutes the basis for our knowledge of the Mexican terrestrial arthropods. This monumental contribution lists about 35,000 species of Mexican and Central American terrestrial arthropods (Llorente Bousquets et al. 1996b). Llorente Bousquets et al. (1996a, 2000, 2004), Llorente Bousquets \& Morrone (2002), and Morrone \& Llorente Bousquets (2006) compiled the most recent information on Mexican arthropods, contained in five volumes and more than 3,800 pages, where several authors provided synthetic treatments of a vast array of Mexican arthropod taxa. The numbers that can be obtained from the chapters of these books are detailed in Table I. There are about 38,000 species of described Mexican arachnids and hexapods, although several taxa have not been reviewed yet.

Llorente Bousquets et al. (1996b) estimated that the number of Mexican species of arachnids and hexapods ranges from 300,000 to 700,000, depending on the method employed. According to these authors, Mexico concentrates $10 \%$ of the species of the world, being more than $10 \%$ of them endemic to the country.

\section{HALFFTER'S MEXICAN MONTANE BIOGEOGRAPHIC PATTERNS}

Halffter (1962, 1965, 1972, 1974, 1976, 1978, 1987, 2003) analyzed distributional patterns of Scarabaeidae (Coleoptera) taxa in the Mexican Transition Zone, categorizing them as three distributional patterns. In addition to their distributions in the area, Halffter took into consideration where their closest relatives were found, the degree of species sympatry, and the diversity of habitats occupied by the species (Liebherr 1991, 1994). 
The Nearctic distributional pattern includes taxa that underwent diversification in the mountains of Mexico during Pliocene and Pleistocene. Their closest relatives are found further north along the Rocky Mountain Cordillera and areas across North America north of Mexico. The Isthmus of Tehuantepec constitutes its southern limit, but there may be a few species in Central America. Taxa belonging to this pattern usually occupy temperate conifer forests and grasslands found above $1700 \mathrm{~m}$ of elevation.

The Paleoamerican distributional pattern includes taxa that underwent diversification prior to the Pliocene closure of the Isthmus of Tehuantepec. Their closest relatives are Old World temperate and tropical taxa. They are restricted to Mexican mountain areas, with ecological preferences for deserts, grasslands, and rain forests, and may also have some species in Central America.

The Mesoamerican distributional pattern includes taxa that evolved in situ in humid montane habitats throughout Mexico and Central America. They have their highest species diversity in Central America, with species in the tropical and cloud forests in the mountains of Oaxaca, and further north and west along the Atlantic and Pacific versants. They have South American affinities, and are hypothesized to have diversified earlier than the taxa assigned to the Nearctic pattern, in the Oligocene.

\section{A BIOGEOGRAPHICAL PUZZLE}

In order to provide an explanation - from a historical biogeographic viewpointfor the Mexican high arthropod diversity, we provide herein three sets of primary biogeographic homology statements (sensu Morrone 2001b, 2004c), in terms of biogeographic regions, dominions, and provinces. This "puzzle" of increasingly smaller pieces is intended to integrate previous knowledge (Halffter 1972, 1974, 1976, 1978, 1987, 2003; Liebherr 1991, 1994; Llorente Bousquets 1996; Morrone et al. 1999, 2002; Espinosa et al. 2000; Marshall \& Liebherr 2000; Morrone 2001a, 2004c, 2005, 2006; Morrone \& Márquez 2001, 2003; Morrone \& LlorenteBousquets, 2006). In addition, we identify herein some panbiogeographic nodes, in the areas of contact between different biogeographic provinces.

Regions. Two biogeographic regions have been traditionally recognized in Mexico (Sclater 1858; Wallace 1876; Cabrera \& Willink 1973; Morrone et al. 1999, 2002; Morrone 2001a). The Nearctic region basically comprises the Baja California peninsula, Sonora, the Mexican plateau, and Tamaulipas, whereas the Neotropical region comprises the rest of the country. Several analyses have shown that some Neotropical taxa extend further to the north (Morrone 2001a; Morrone \& Márquez 2001, 2003) and a Mexican Transition Zone has been recognized (Darlington 1966; 
Halffter 1962, 1965, 1972, 1974, 1976, 1978, 1987; Morrone 2005, 2006) (Fig. 1). Due to their interconnection, via the Sierra Madre Occidental and the Sierra Madre Oriental that extend into the Nearctic to the north, the mixture of Nearctic and Neotropical biotic elements in the Mexican Transition Zone is strongly favored.

Dominions. Within both biogeographic regions, five dominions have been recognized (Fig. 2), based on the overlap of the distributional patterns of several plant and animal taxa (Morrone et al. 1999; Espinosa et al. 2000; Morrone \& Márquez 2001, 2003). The Californian Nearctic dominion includes the Baja California peninsula, extending further to the north into California (USA). The Continental Nearctic dominion extends in Sonora, the Mexican plateau and Tamaulipas, also extending to the north into the USA. The Mexican Mountain dominion, equivalent to the Transition Zone, is the only dominion restricted to Mexico and represents the place where there is a higher mixture of Nearctic and Neotropical biotic elements. This mixture is not only latitudinal, but also altitudinal, with a major Nearctic influence at higher altitudes and a higher Neotropical influence at lower altitudes. The Antillean dominion extends mainly in the Antilles; in Mexico is found only in the Yucatan peninsula. The Mesoamerican dominion extends into Central America, the Sierra Madre de Chiapas, and the lowlands of the Mexican Gulf and the coast of the Pacific Ocean; taxa belonging to this dominion have been basically assigned to the Mountain Mesoamerican pattern of Halffter (1987).

Provinces. Finally, 14 smaller units or biogeographic provinces (Fig. 3) have been delimited (Espinosa et al. 2000; Morrone 2001 a; Morrone et al. 2002; Morrone, 2005; Morrone \& Llorente-Bousquets, 2006). The California province occupies the northern portion of the Baja California peninsula, from the Sierras of San Pedro Mártir and Juárez, extending northward along the Sierra Nevada into southwestern USA. The Baja California province extends in the rest of Baja California peninsula. The Sonora province extends in the coastal areas in northwestern Mexico, from the northeastern portion of the Baja California peninsula to the Piaxtla river basin in the south. The Mexican Plateau province extends in central Mexico, in the states of Zacatecas, San Luis Potosí, Guanajuato, Chihuahua, Coahuila, Durango, and Nuevo Mexico, as well as small parts of Nuevo León and Sonora, below 4,000 m altitude. The Tamaulipas province occupies coastal areas in the northern part of the Mexican Gulf, north of the Pánuco river basin. The Sierra Madre Occidental province extends in western Mexico, in the states of Chihuahua, Durango, Zacatecas, Sonora, Sinaloa, Nayarit, and Jalisco, above 1,000 m altitude. The Sierra Madre Oriental province extends in eastern Mexico, in the states of San Luis Potosí, Coahuila, Hidalgo, Nuevo León, and Querétaro, above 1,500 m altitude. The Transmexican Volcanic Belt province occupies central Mexico, in the states of Guanajuato, 
Mexico, Distrito Federal, Jalisco, Michoacán, Puebla, Oaxaca, Tlaxcala, and Veracruz. The Balsas Basin province extends in central Mexico, in the states of Guerrero, Mexico, Jalisco, Michoacán, Morelos, Oaxaca, and Puebla, below 2,000 $m$ altitude. The Sierra Madre del Sur province extends in south central Mexico, from southern Michoacán to Guerrero and Oaxaca, and part of Puebla, above 1,000 m altitude. The Yucatán peninsula province corresponds to the states of Campeche, Yucatán, and Quintana Roo, below 200 m altitude. The Mexican Gulf province corresponds to the coast of the Mexican Gulf, in eastern Mexico, Belize, and northern Guatemala. The Mexican Pacific Coast province occupies western Mexico, in the Pacific coast of the states of Sinaloa, Nayarit, Colima, Jalisco, Michoacán, Guerrero, Oaxaca, and Chiapas. The Chiapas province extends in southern Mexico, Guatemala, and Nicaragua, basically corresponding to the Sierra Madre de Chiapas, from 500 to $2,000 \mathrm{~m}$ altitude.

Nodes. We assumed that the Mexican biogeographic provinces do represent generalized tracks and that the areas where at least three biogeographic provinces intersect can be considered preliminarily as panbiogeographic nodes. These areas have a high diversity due to contact between different biotic elements (Craw et al. 1999) and should have priority for biodiversity conservation (Morrone \& Espinosa 1998; Morrone 1999, 2004c; Luna et al., 2000; Contreras-Medina et al. 2001). These fifteen nodes (Fig. 4) are the following (the corresponding biogeographic provinces are indicated between brackets): California Norte (Baja California, California, and Sonora), Sonora (Sonora, Sierra Madre Occidental, and Mexican Plateau), SonoraChihuahua-Sinaloa (Sonora, Sierra Madre Occidental, and Pacific Coast), JaliscoNayarit (Sierra Madre Occidental, Transmexican Volcanic Belt, and Mexican Pacific Coast), Jalisco-Zacatecas (Sierra Madre Occidental, Transmexican Volcanic Belt, and Mexican Plateau), Monterrey, Nuevo León (Tamaulipas, Mexican Plateau, and Sierra Madre Oriental), Ciudad Victoria, Tamaulipas (Tamaulipas, Sierra Madre Oriental, and Mexican Gulf), northwestern Michoacán (Transmexican Volcanic Belt, Balsas Basin, and Mexican Pacific Coast), northern Guerrero (Mexican Pacific Coast, Balsas Basin, and Sierra Madre del Sur), Hidalgo-Puebla (Mexican Plateau, Sierra Madre Oriental, and Transmexican Volcanic Belt), Orizaba-Córdoba, Veracruz (Sierra Madre Oriental, Transmexican Volcanic Belt, and Mexican Gulf), Huajuapan de León, Oaxaca (Balsas Basin, Transmexican Volcanic Belt, and Sierra Madre del Sur), Sierra de Juárez, Oaxaca (Mexican Volcanic Belt, Sierra Madre del Sur, and Mexican Gulf), southern Oaxaca (Sierra Madre del Sur, Mexican Pacific Coast, and Mexican Gulf), and El Ocote, Chiapas (Mexican Gulf, Mexican Pacific Coast, and Chiapas). The biggest concentration of nodes is found toward the eastern and western extremes and the center of the country, where the main mountainous chains are located. 


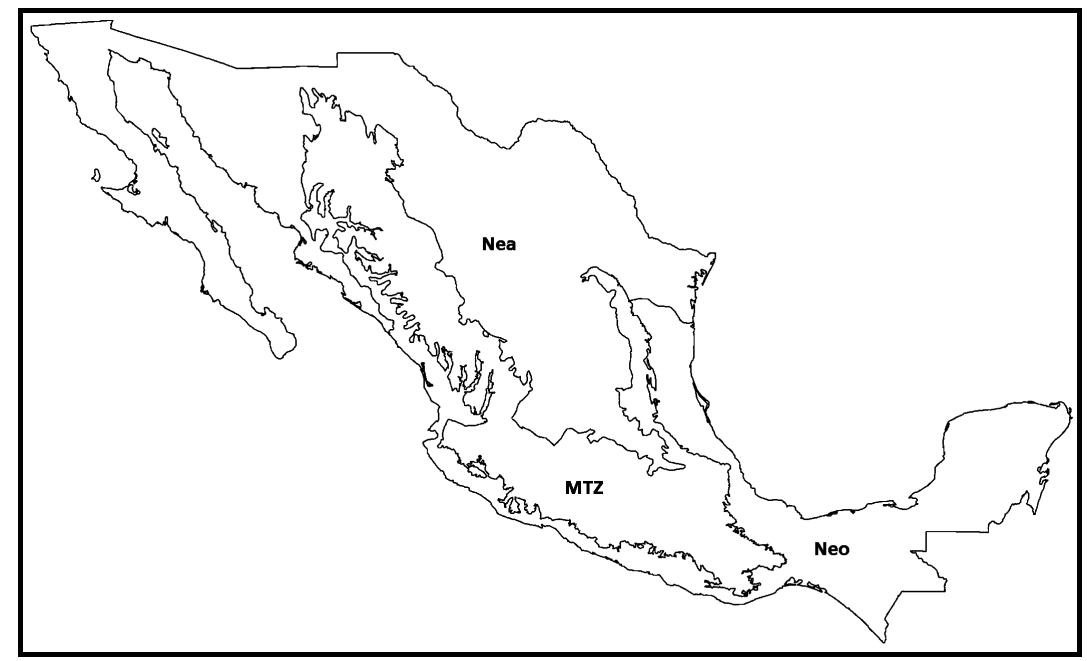

Figure 1

Biogeographic regions and Mexican Transition Zone. MTZ: Mexican Transition Zone, Nea: Nearctic region, Neo: Neotropical region.

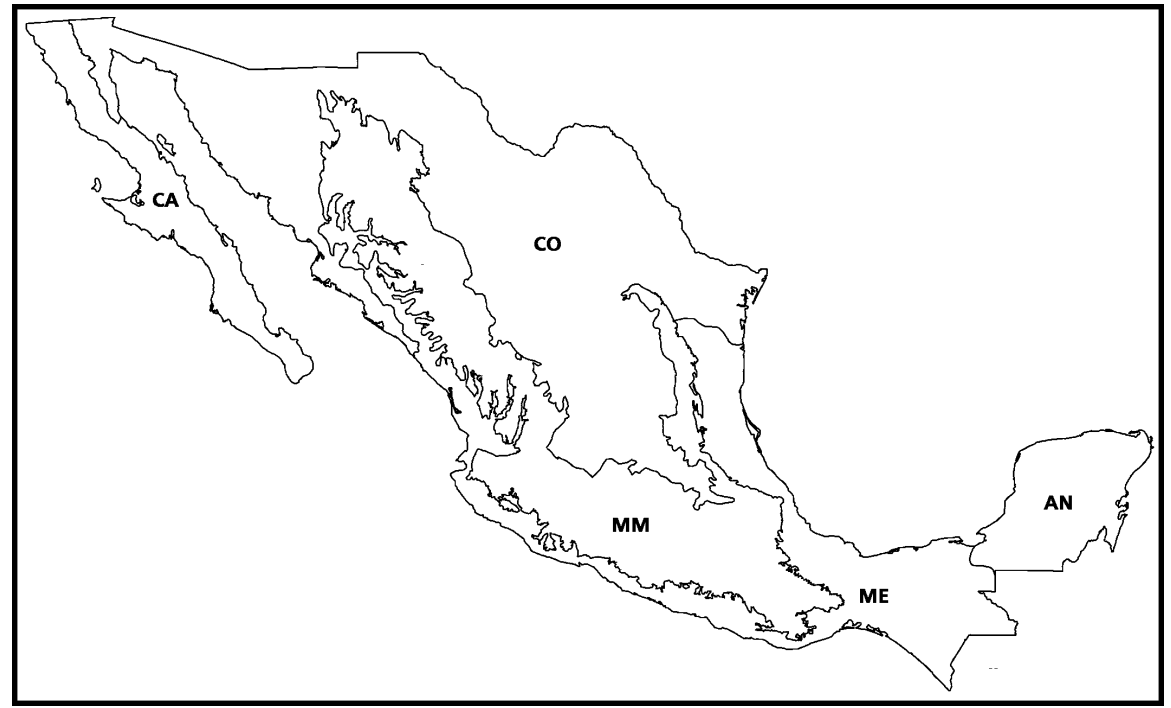

Figure 2

Biogeographic dominions. AN: Antillean, CA: Californian Nearctic, CO: Continental Nearctic, ME: Mesoamerican, MM: Mexican Mountain (= Mexican Transition Zone). 


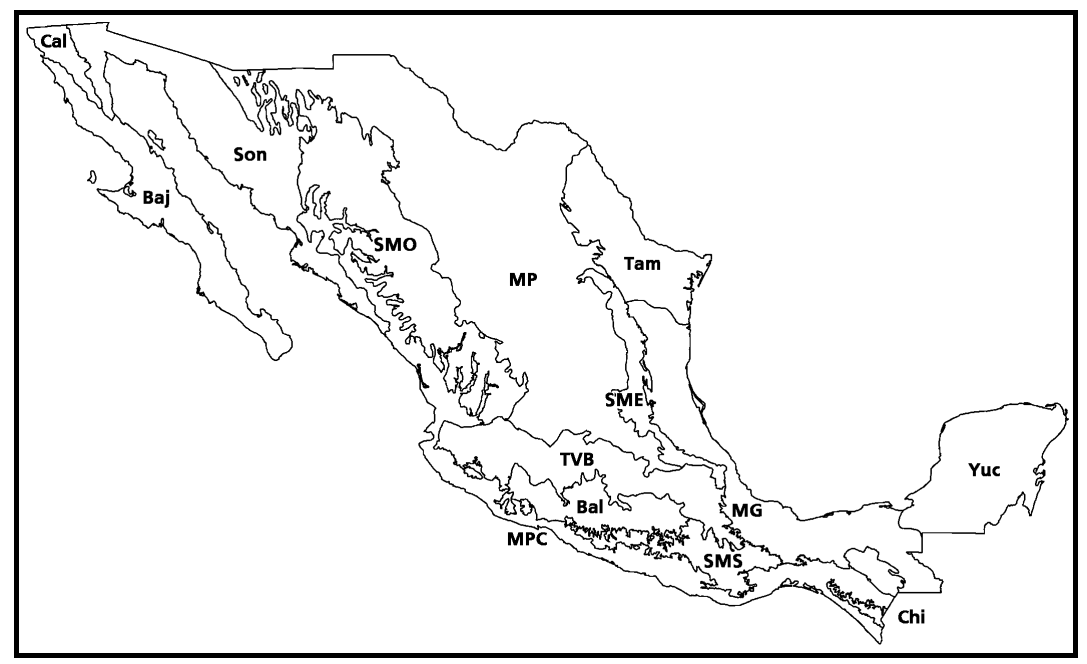

Figure 3

Biogeographic provinces. Baj: Baja California, Bal: Balsas Basin, Cal: California, Chi: Chiapas, MG: Mexican Gulf, MP: Mexican Plateau, MPC: Mexican Pacific Coast, SME: Sierra Madre Oriental, SMO: Sierra Madre Occidental, SMS: Sierra Madre del Sur, Son: Sonora, Tam: Tamaulipas, TVB: Transmexican Volcanic Belt, Yuc: Yucatán.

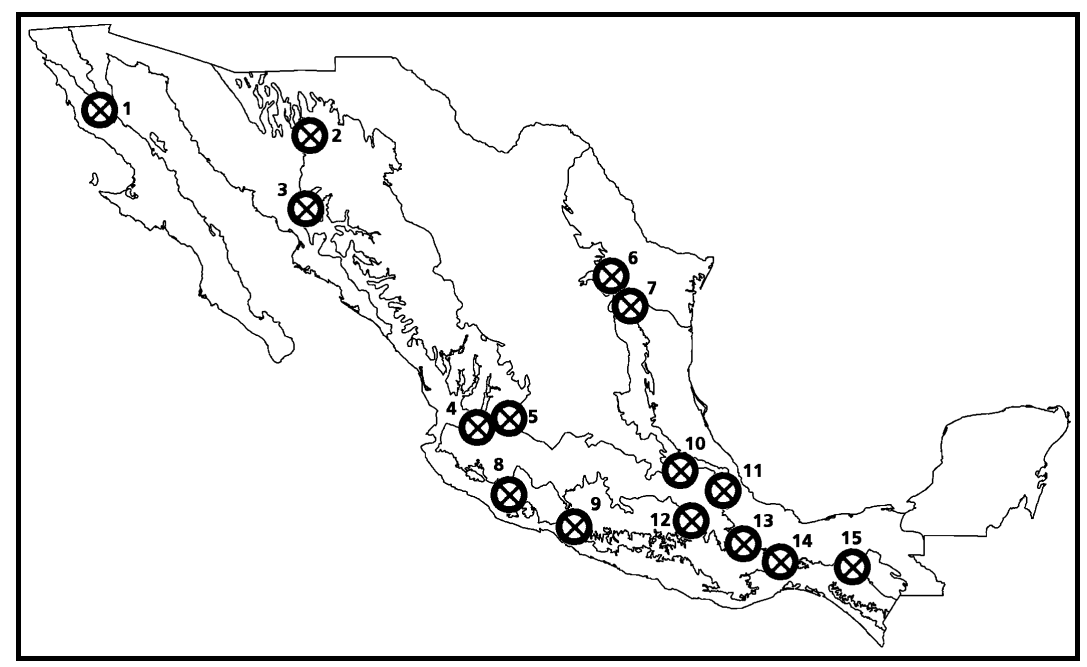

Figure 4

Panbiogeographic nodes. 1: Baja California Norte, 2: Sonora, 3: Sonora-Chihuahua-Sinaloa, 4: JaliscoNayarit, 5: Jalisco-Zacatecas, 6: Monterrey, 7: Ciudad Victoria, 8: northwestern Michoacán, 9: northern Guerrero, 10: Hidalgo-Puebla, 11: Orizaba-Córdoba, 12: Huajuapan de León, 13: Sierra de Juárez, 14: southern Oaxaca, and 15: El Ocote. 


\section{Table I}

Number of Mexican species of arachnids and hexapods, with indication of endemics and literature. Arachnid orders arranged phylogenetically following Dunlop (2002) and Hexapod orders arranged phylogenetically following Carpenter y Wheeler (1999).

\begin{tabular}{|c|c|c|c|c|c|}
\hline & $\begin{array}{l}\text { Mexican } \\
\text { species }\end{array}$ & $\begin{array}{c}\% \\
\text { of the } \\
\text { world }\end{array}$ & $\begin{array}{l}\text { Endemic } \\
\text { species }\end{array}$ & $\begin{array}{c}\text { \% of } \\
\text { species } \\
\text { endemic }\end{array}$ & Literature \\
\hline \multicolumn{6}{|l|}{ ARACHNIDA } \\
\hline Scorpiones & 177 & 11.8 & 162 & 91.5 & Lourenço \& Sissom (2000) \\
\hline Opiliones & 283 & 4.6 & 218 & 77 & Kury \& Cokendolpher (2000) \\
\hline Solifugae & 57 & - & - & - & Vázquez Rojas (1996a) \\
\hline Acari & 2343 & & & & \\
\hline --Oribatei & 434 & 4.6 & - & - & $\begin{array}{l}\text { Hoffmann \& López-Campos (2002) } \\
\text { Palacios-Vargas \& Iglesias (2004) }\end{array}$ \\
\hline Ricinulei & 10 & 20.4 & - & - & Vázquez Rojas (1996b) \\
\hline Palpigradi & 1 & 2.1 & 1 & 100 & Vázquez Rojas (1996c) \\
\hline Araneae & 2506 & 7.3 & 1759 & 70.2 & Jiménez (1996) \\
\hline Amblypygi & 14 & 20 & - & - & Vázquez Rojas (1996d) \\
\hline Uropygi & 2 & 2.3 & 0 & 0 & Vázquez Rojas (1996e) \\
\hline Schizomida & 35 & - & - & - & Vázquez Rojas (1996f) \\
\hline Pseudoscorpionida & a 151 & 4.7 & 110 & 72.8 & Ceballos (2004) \\
\hline \multicolumn{6}{|l|}{ HEXAPODA } \\
\hline Diplura & 48 & - & - & - & Palacios-Vargas (2000a) \\
\hline Collembola & 550 & 7.8 & - & - & Palacios-Vargas et al. (2000) \\
\hline Protura & 14 & 7 & - & - & Palacios-Vargas (2000a) \\
\hline Archaeognatha & 14 & - & - & - & Palacios-Vargas (2000b) \\
\hline Zygentoma & 36 & - & - & - & Palacios-Vargas (2000b) \\
\hline Ephemeroptera & 116 & 4.6 & $>30$ & $>25.9$ & McCafferty \& Lugo-Ortiz (1996) \\
\hline Odonata & 352 & 6.3 & 40 & 11.4 & González Soriano \& Novelo Gutiérrez (1996) \\
\hline Plecoptera & 47 & 2.6 & - & - & Baumann \& Kondratieff (1996) \\
\hline Embioptera & 37 & 12.8 & 32 & 86.4 & Szumik (2002) \\
\hline Isoptera & 63 & 2.3 & - & - & Cancello \& Myles (2000) \\
\hline Orthoptera & 920 & 7.7 & - & - & Barrientos-Lozano (2004) \\
\hline Dermaptera & 51 & 2.5 & 16 & 31.4 & Sakai (2004) \\
\hline Zoraptera & 1 & - & 1 & 100 & Engel (2004) \\
\hline \multicolumn{6}{|l|}{ HEMIPTERA } \\
\hline Enicocephalidae & 12 & 3.0 & - & - & O'Brien et al. (1996), \\
\hline Lygaeidae & 284 & 5.7 & - & - & Slater \& Brailovsky (2000), \\
\hline Pentatomidae & 317 & - & - & - & Thomas (2000), Coscarón (2002), and \\
\hline Reduviidae & 248 & 3.8 & - & - & Coscarón \& Dellapé (2002) \\
\hline Homoptera & 2773 & - & & & \\
\hline --Membracidae & & & 105 & 4 & \\
\hline --Fulgoridae & & & 23 & 6 & \\
\hline --Aleyrodidae & & & 42 & 63 & \\
\hline --Coccoidea & & & 190 & 38 & \\
\hline
\end{tabular}


Morrone \& Márquez: Biodiversity of mexican terrestrial arthropods

Tabla 1. Continuation

\begin{tabular}{|c|c|c|c|c|c|}
\hline & $\begin{array}{c}\text { Mexican } \\
\text { species }\end{array}$ & $\begin{array}{c}\% \\
\text { of the } \\
\text { world }\end{array}$ & $\begin{array}{c}\text { Endemic } \\
\text { species }\end{array}$ & $\begin{array}{c}\text { \% of } \\
\text { species } \\
\text { endemic }\end{array}$ & Literature \\
\hline Thysanoptera & 600 & 12.0 & & & Johansen \& Mojica-Guzmán (1996) \\
\hline --Terebrantia & & & 200 & 62.5 & \\
\hline --Tubulifera & & & 194 & 69.2 & \\
\hline Psocoptera & 642 & 7.9 & 475 & 74 & Mockford \& García Aldrete (1996) \\
\hline \multicolumn{6}{|l|}{ COLEOPTERA } \\
\hline Agyrtidae & 3 & 5.2 & 3 & 100 & \multirow{29}{*}{$\begin{array}{l}\text { Anderson \& O'Brien (1996) } \\
\text { Hespenheide (1996), Morón (1996a, b } \\
\text { 2006a, b), Navarrete Heredia \& Newtor } \\
\text { (1996, 2002), Noguera \& Chemsack } \\
\text { (1996), Zaragoza Caballero \& Mendoza } \\
\text { Ramírez (1996), Ball \& Shpeley (2000) } \\
\text { Navarrete-Heredia \& Burgos-Soloric } \\
\text { (2000), Navarrete-Heredia \& Fierros- } \\
\text { López (2000), Peck (2000), Santiago } \\
\text { Fragoso \& Spangler (2000), Aalbu et al } \\
\text { (2002), Equihua Martínez \& Burgo } \\
\text { Solorio (2002), Morrone (2002, 2004a } \\
\text { b), Reyes-Castillo (2002a, b), Romero } \\
\text { Nápoles (2002), Navarrete-Heredia \& } \\
\text { Quiroz-Rocha (2004), Navarrete-Heredia } \\
\text { et al. (2004a, b), Furth (2004), Alonso- } \\
\text { Zarazaga (2004), Morrone \& Cuevas } \\
\text { (2004), Corona \& Toledo (2006), Cueva } \\
\text { (2006), Márquez \& Asiain (2006) } \\
\text { Navarrete-Heredia \& Beutel (2006) } \\
\text { Ordóñez Reséndiz (2006), Ordóñez } \\
\text { Reséndiz \& Eligio García (2006), Reyes- } \\
\text { Castillo et al. (2006), Toledo \& Corona } \\
\text { (2006), and Zaragoza Caballero et al } \\
\text { (2006). }\end{array}$} \\
\hline Apionidae & 170 & - & - & - & \\
\hline Belidae & 2 & - & 2 & 100 & \\
\hline Buprestidae & 863 & - & - & - & \\
\hline Cantharidae & 178 & 5.1 & 115 & 64.5 & \\
\hline Carabidae & 1042 & - & 1191 & 60 & \\
\hline Cerambycidae & 1605 & - & 787 & 56.9 & \\
\hline Chrysomelidae & 1831 & - & - & - & \\
\hline Ciidae & 42 & $7-6$ & - & - & \\
\hline Curculionidae & 3190 & 4.68 & 951 & 28 & \\
\hline Dryophthoridae & 125 & - & 29 & 22.8 & \\
\hline Elmidae & 64 & 5.3 & 1 & 1.5 & \\
\hline Erirhinidae & 21 & 8.3 & 3 & 14.2 & \\
\hline Erotylidae & 123 & 4.9 & 43 & 34.9 & \\
\hline Histeridae & 281 & 7.2 & 71 & 25.2 & \\
\hline Hydraenidae & 71 & 5.9 & 36 & 50.7 & \\
\hline Lampyridae & 91 & 4.8 & 53 & 58.2 & \\
\hline Leiodidae & 87 & 3.0 & 72 & 82.7 & \\
\hline Lepiceridae & 2 & 100 & 1 & 50 & \\
\hline Lucanidae & 4 & 0.3 & 2 & 50 & \\
\hline Lycidae & 154 & - & - & - & \\
\hline Melolonthidae & 1147 & - & 671 & - & \\
\hline Nemonychidae & 2 & 2.9 & 2 & 100 & \\
\hline Passalidae & 83 & 12.0 & 43 & 52.4 & \\
\hline Phengodidae & 45 & 21.1 & 31 & 68.8 & \\
\hline Scarabaeidae & 461 & - & 204 & - & \\
\hline Silphidae & 11 & 5.8 & 1 & 9.1 & \\
\hline Staphylinidae & 1487 & - & - & - & \\
\hline Tenebrionidae & 1227 & 6.4 & 712 & 58.0 & \\
\hline Neuroptera & 311 & 5.2 & - & - & Oswald et al. (2002) \\
\hline Megaloptera & 13 & 4.3 & - & - & Contreras-Ramos (2000) \\
\hline Raphidioptera & 13 & 6.5 & 8 & 61.5 & Aspöck \& Aspöck (1996) \\
\hline \multicolumn{6}{|c|}{ HYMENOPTERA } \\
\hline Aphelinidae & 95 & 8.4 & 7 & 7.3 & Ayala et al. (1996), Rodríguez Palafox \\
\hline Apoidea & 1800 & 9.0 & - & - & (1996), Rojas Fernández (1996), \\
\hline Braconidae & 355 & 2.4 & - & - & González-Hernández (2000), Wharton \& \\
\hline
\end{tabular}


Tabla 1. Conclusion

\begin{tabular}{|c|c|c|c|c|c|}
\hline & $\begin{array}{l}\text { Mexican } \\
\text { species }\end{array}$ & $\begin{array}{c}\% \\
\text { of the } \\
\text { world }\end{array}$ & $\begin{array}{l}\text { Endemic } \\
\text { species }\end{array}$ & $\begin{array}{c}\text { \% of } \\
\text { species } \\
\text { endemic }\end{array}$ & Literature \\
\hline Chalcidoidea & 908 & 1.5 & - & - & \multirow{11}{*}{$\begin{array}{l}\text { Mercado (2000), Díaz \& Gallardo } \\
\text { (2002), Loiácono \& Margaría (2002), } \\
\text { Ruiz Cancino et al. (2002a, b, 2004), } \\
\text { Ruiz Cancino \& Coronado Blanco } \\
\text { (2002), Coronado Blanco et al. (2004), } \\
\text { Kasparyan \& Ruiz Cancino (2004), } \\
\text { Triapitsyn et al. (2004a, b), Fursov et al. } \\
\text { (2004), Myartseva et al. (2004), and } \\
\text { Yánez-Ordóñez \& Trujano Ortega } \\
\text { (2006). }\end{array}$} \\
\hline Cynipoidea & 168 & 5.6 & - & - & \\
\hline Encyrtidae & 164 & 4.3 & 34 & 21 & \\
\hline Formicidae & 501 & 5.7 & 112 & 22.3 & \\
\hline Ichneumonidae & 948 & 0.9 & 474 & 50.0 & \\
\hline Mymaridae & 47 & 3.4 & 7 & 14.9 & \\
\hline Pompiloidea & 174 & 4.1 & - & - & \\
\hline Proctotrupoidea & 95 & 1.8 & - & - & \\
\hline Sphecoidea & 615 & 7.7 & 157 & 25.0 & \\
\hline Trichogrammatida & ae 47 & 7.2 & 2 & 4.2 & \\
\hline Vespidae & 346 & 8.3 & 82 & 26.0 & \\
\hline Trichoptera & 325 & 3.0 & - & - & Bueno Soria (1996) \\
\hline \multicolumn{6}{|l|}{ LEPIDOPTERA } \\
\hline Acrolophidae & 53 & 19.8 & - & - & \multirow{12}{*}{$\begin{array}{l}\text { Llorente Bousquets et al. (1996c, 2006), } \\
\text { Razowski (1996), Solís (1996), Balcázar } \\
\text { Lara \& Beutelspacher Baigts (2000a, b), } \\
\text { Becker (2000), Davis (2000), León- } \\
\text { Cortés (2000), Miller (2000), Warren } \\
\text { (2000), León-Cortés et al. (2006), } \\
\text { Balcázar Lara (2006), Luis-Martínez et } \\
\text { al. (2006), Oñate-Ocaña et al. (2006), } \\
\text { and Vargas-Fernández et al. (2006). }\end{array}$} \\
\hline Arctiidae & 418 & 6.0 & 158 & 38.0 & \\
\hline Castniidae & 14 & 11.0 & 8 & 57.0 & \\
\hline Gracillaridae & 13 & 0.6 & - & - & \\
\hline Hesperioidea & 741 & - & 150 & 20.2 & \\
\hline Microlepidoptera & 2000 & - & - & - & \\
\hline Papilionoidea & 2000 & 10.0 & - & - & \\
\hline Pyraloidea & 409 & 1.3 & - & - & \\
\hline Saturniidae & 195 & 16.0 & 88 & 45.3 & \\
\hline Sphingoidea & 195 & 18.0 & 17 & 8.7 & \\
\hline Tineoidae & 26 & 1.0 & - & - & \\
\hline Tortricidae & 441 & - & - & - & \\
\hline Siphonaptera & 142 & 5.6 & - & & $\begin{array}{l}\text { Ponce Ulloa \& Llorente Bousquets (1996) } \\
\text { and Gutiérrez-Velázquez et al. (2006) }\end{array}$ \\
\hline Mecoptera & 9 & - & 8 & 88.8 & Byers (1996) \\
\hline \multicolumn{6}{|l|}{ DIPTERA } \\
\hline Bibionidae & 40 & 6.1 & 18 & 45.0 & \multirow{11}{*}{$\begin{array}{l}\text { Hernández-Ortiz (1996), Ibáñez-Bernal } \\
\text { \& Coscarón (1996, 2000), Ibáñez-Bernal } \\
\text { et al. (1996a, b, 2004), Papavero (1996), } \\
\text { Andersen et al. (2000), Contreras-Ramos } \\
\text { \& Gelhaus (2002), Fitzgerald (2000), and } \\
\text { Ibáñez-Bernal (2000). }\end{array}$} \\
\hline Ceratopogonidae & 168 & - & 23 & 13.6 & \\
\hline Chironomidae & 61 & 1.0 & - & - & \\
\hline Culicidae & 247 & 7.2 & 22 & 8.9 & \\
\hline Dolichopodidae & 203 & 3.1 & 101 & 49.7 & \\
\hline Mydidae & 38 & - & - & - & \\
\hline Psychodidae & 65 & - & - & - & \\
\hline Simuliidae & 90 & 6.1 & - & - & \\
\hline Tabanidae & 201 & 4.7 & 101 & 50.0 & \\
\hline Tephritidae & 224 & 5.3 & 191 & 85.2 & \\
\hline Tipulidae & 324 & 2.1 & 259 & 80.0 & \\
\hline
\end{tabular}




\section{CONCLUSIONS}

There are still a lot of taxa of Mexican terrestrial arthropods for which knowledge is poor or even absent. Llorente Bousquets et al. (1996b) concluded that the country lacks specialists for the majority of the Arthropod families, and that about 300 taxonomists would be necessary to study the poorly known taxa. The "puzzle" of primary biogeographic homology statements herein outlined leads to recognize two regions, one transition zone, five dominions, and 14 provinces. The Mexican Transition Zone presents the highest level of biotic mixture between Nearctic and Neotropical elements. Species distributed in the lowlands near the Mexican coasts have mostly Neotropical affinities, and can be detected further north than previously hypothesized.

Biogeographic nodes possess a higher biodiversity, with different biogeographic affinities. Some of these areas are currently under protection for their high biodiversity, as the Biological Reserves "El Ocote" (Chiapas) and "El Cielo" (Tamaulipas). Others have been studied and characterized as highly biodiverse, as Orizaba and Córdoba (Toledo 1981), Sierra de Juárez (Luis et al. 1991), northern Guerrero (Luna \& Llorente 1993), etc. None of them has been studied adequately, and future research will be very important to select more sites with high biodiversity, and species representing different lineages and biogeographic histories.

Cladistic biogeographic analyses based on taxon area cladograms -secondary biogeographic homology sensu Morrone (2001b, 2004c)- will allow us to falsify these hypotheses. Such analyses will corroborate some of these hypotheses and will also refine them, e.g., by providing the sequence of area fragmentations involved. In addition, geological or geotectonic analyses will also allow testing the hypotheses herein outlined.

\section{ACKNOWLEDGMENTS}

We thank critical comments by two anonymous reviewers.

\section{REFERENCES}

Aalbu, R.L., G.E. Flores \& C.A. Triplehorn. 2002. Tenebrionidae. Pp. 499-512. In: J. Llorente Bousquets and J. J. Morrone (Eds.). Biodiversidad, taxonomía y biogeografia de artrópodos de México: Hacia una síntesis de su conocimiento, Vol. III. Facultad de Ciencias, UNAM, Mexico, D.F.

Alonso-Zarazaga, M.A. 2004. Apionidae (Coleoptera). Pp. 691-699. In: J. Llorente Bousquets, J.J. Morrone, O. Yáñez-Ordóñez and I. Vargas Fernández (Eds.). Biodiversidad, taxonomía y biogeografía de artrópodos de México: Hacia una síntesis de su conocimiento, Vol. IV. Facultad de Ciencias, UNAM, Mexico, D.F. 
Andersen, T., A. Contreras-Ramos \& M. Spies. 2000. Chironomidae (Diptera). Pp. 581591. In: J. Llorente Bousquets, E. González and N. Papavero (Eds.). Biodiversidad, taxonomía y biogeografía de artrópodos de México: Hacia una síntesis de su conocimiento, Vol. II. Facultad de Ciencias, UNAM, Mexico, D.F.

Anderson, R.S. \& C.W. O'Brien. 1996. Curculionidae (Coleoptera). Pp. 329-351. In: J. Llorente Bousquets, A.N. García Aldrete and E. González Soriano (Eds.). Biodiversidad, taxonomía y biogeografía de artrópodos de México: Hacia una sintesis de su conocimiento. Instituto de Biología, UNAM, Mexico, D.F.

Aspöck, U. \& H. Aspöck. 1996. Raphidioptera. Pp. 277-286. In: J. Llorente Bousquets, A.N. García Aldrete and E. González Soriano (Eds.). Biodiversidad, taxonomía y biogeografía de artrópodos de México: Hacia una sintesis de su conocimiento. Instituto de Biología, UNAM, Mexico, D.F.

Ayala, R., T.L. Griswold \& D. Yanega. 1996. Apoidea (Hymenoptera). Pp. 423-464. In: J. Llorente Bousquets, A.N. García Aldrete and E. González Soriano (Eds.). Biodiversidad, taxonomía y biogeografía de artrópodos de México: Hacia una sintesis de su conocimiento. Instituto de Biología, UNAM, Mexico, D.F.

Balcázar Lara, M.A. 2006. Patrones de distribución de la familia Saturniidae (Lepidoptera). Pp. 649-659. In: J.J. Morrone and J. Llorente Bousquets (Eds.). Componentes bióticos principales de la entomofauna mexicana. Vols. I y II. Las Prensas de Ciencias, UNAM, Mexico, D.F.

Balcázar Lara, M.A. \& C.R. Beutelspacher Baigts. 2000a. Saturniidae (Lepidoptera). Pp. 501-513. In: J. Llorente Bousquets, E. González and N. Papavero (Eds.). Biodiversidad, taxonomía y biogeografía de artrópodos de México: Hacia una síntesis de su conocimiento, Vol. II. Facultad de Ciencias, UNAM, Mexico, D.F.

Balcázar Lara, M.A. \& C.R. Beutelspacher Baigts. 2000b. Arctiidae: Lithosiinae, Arctiinae, Pericopinae (Lepidoptera). Pp. 515-525. In: J. Llorente Bousquets, E. González and N. Papavero (Eds.). Biodiversidad, taxonomía y biogeografía de artrópodos de México: Hacia una sintesis de su conocimiento, Vol. II. Facultad de Ciencias, UNAM, Mexico, D.F.

Ball, G.E. \& D. Shpeley. 2000. Carabidae (Coleoptera). Pp. 363-399. In: J. Llorente Bousquets, E. González and N. Papavero (Eds.). Biodiversidad, taxonomía y biogeografía de artrópodos de México: Hacia una síntesis de su conocimiento, Vol. II. Facultad de Ciencias, UNAM, Mexico, D.F.

Barrientos-Lozano, L. 2004. Orthoptera. Pp. 604-625. In: J. Llorente Bousquets, J.J. Morrone, O. Yáñez-Ordóñez and I. Vargas Fernández (Eds.). Biodiversidad, taxonomía y biogeografía de artrópodos de México: Hacia una síntesis de su conocimiento, Vol. IV. Facultad de Ciencias, UNAM, Mexico, D.F.

Baumann, R. W. \& B.C. Kondratieff. 1996. Plecoptera. Pp. 169-174. In: J. Llorente Bousquets, A.N. García Aldrete and E. González Soriano (Eds.). Biodiversidad, taxonomía y biogeografía de artrópodos de México: Hacia una síntesis de su conocimiento. Instituto de Biología, UNAM, Mexico, D.F.

Becker, V.O. 2000. Microlepidoptera. Pp. 453-468. In: J. Llorente Bousquets, E. González and N. Papavero (Eds.). Biodiversidad, taxonomía y biogeografía de artrópodos de 
Morrone \& Márquez: Biodiversity of mexican terrestrial arthropods

México: Hacia una síntesis de su conocimiento, Vol. II. Facultad de Ciencias, UNAM, Mexico, D.F.

Bueno Soria, J. 1996. Trichoptera. Pp. 501-511. In: J. Llorente Bousquets, A.N. García Aldrete and E. González Soriano (Eds.). Biodiversidad, taxonomía y biogeografía de artrópodos de México: Hacia una síntesis de su conocimiento. Instituto de Biología, UNAM, Mexico, D.F.

Byers, G.W. 1996. Mecoptera. Pp. 549-552. In: J. Llorente Bousquets, A.N. García Aldrete and E. González Soriano (Eds.). Biodiversidad, taxonomía y biogeografía de artrópodos de México: Hacia una síntesis de su conocimiento. Instituto de Biología, UNAM, Mexico, D.F.

Cabrera, A.L. \& A. Willink. 1973. Biogeografía de América Latina. Monografía 13, Serie de Biología, OEA, Washington, D. C.

Cancello, E.M. \& T.G. Myles. 2000. Isoptera. Pp. 295-315. In: J. Llorente Bousquets, E. González and N. Papavero (Eds.). Biodiversidad, taxonomía y biogeografía de artrópodos de México: Hacia una sintesis de su conocimiento, Vol. II. Facultad de Ciencias, UNAM, Mexico, D.F.

Carpenter, J.M. \& W. Wheeler. 1999. Cladística numérica, análisis simultáneo y filogenia de los Hexapoda. Pp. 333-346. In: Melic, A., J. J. de Haro, M. Méndez \& I. Ribera (Eds.). Evolución y filogenia de Arthropoda. Boletín de la S.E.A. 26, Zaragoza.

Ceballos, A. 2004. Pseudoscorpionida. Pp. 417-429. In: J. Llorente Bousquets, J.J. Morrone, O. Yáñez-Ordóñez and I. Va rgas Fernández (Eds.). Biodiversidad, taxonomía y biogeografía de artrópodos de México: Hacia una síntesis de su conocimiento, Vol. IV. Facultad de Ciencias, UNAM, Mexico, D.F.

Contreras-Medina, R., J.J. Morrone \& I. Luna-Vega. 2001. Biogeographic methods identify gymnosperm biodiversity hotspots. Naturwissenschaften 88: 427-430.

Contreras-Ramos, A. 2000. Megaloptera (Neuropterida). Pp. 355-362. In: J. Llorente Bousquets, E. González and N. Papavero (Eds.). Biodiversidad, taxonomía y biogeografía de artrópodos de México: Hacia una síntesis de su conocimiento, Vol. II. Facultad de Ciencias, UNAM, Mexico, D.F.

Contreras-Ramos, A. \& J.K. Gelhaus. 2002. Tipulidae. Pp. 583-595. In: J. Llorente Bousquets and J. J. Morrone (Eds.). Biodiversidad, taxonomía y biogeografía de artrópodos de México: Hacia una síntesis de su conocimiento, Vol. III. Facultad de Ciencias, UNAM, Mexico, D.F.

Corona, A.M. \& V.H. Toledo. 2006. Patrones de distribución de la familia Buprestidae (Coleoptera). Pp. 333-391. In: J.J. Morrone and J. Llorente Bousquets (Eds.). Componentes bióticos principales de la entomofauna mexicana. Vols. I y II. Las Prensas de Ciencias, UNAM, Mexico, D.F.

Coronado Blanco, J.M., E. Ruiz Cancino \& S.E. Varela Fuentes. 2004. Adenda a Braconidae (Hymenoptera). Pp. 713-720. In: J. Llorente Bousquets, J.J. Morrone, O. Yáñez-Ordóñez and I. Vargas Fernández (Eds.). Biodiversidad, taxonomía y biogeografía de artrópodos de México: Hacia una síntesis de su conocimiento, Vol. IV. Facultad de Ciencias, UNAM, Mexico, D.F.

Coscarón, M.C. 2002. Reduviidae. Pp. 455-466. In: J. Llorente Bousquets and J. J. Morrone (Eds.). Biodiversidad, taxonomía y biogeografía de artrópodos de México: Hacia una síntesis de su conocimiento, Vol. III. Facultad de Ciencias, UNAM, Mexico, D.F. 
Coscarón, M.C. \& P.M. Dellapé. 2002. Enicocephalomorpha. Pp. 449-454. In: J. Llorente Bousquets and J. J. Morrone (Eds.). Biodiversidad, taxonomía y biogeografia de artrópodos de México: Hacia una síntesis de su conocimiento, Vol. III. Facultad de Ciencias, UNAM, Mexico, D.F.

Craw, R.C., J.R. Grehan \& M.J. Heads. 1999. Panbiogeography: Tracking the history of life. Oxford Biogeography Series 11, Oxford University Press, New York.

Cuevas, P.I. 2006. Patrones de distribución de la familia Dryophthoridae (Coleoptera). Pp. 515-562. In: J.J. Morrone and J. Llorente Bousquets (Eds.). Componentes bióticos principales de la entomofauna mexicana. Vols. I y II. Las Prensas de Ciencias, UNAM, Mexico, D.F.

Darlington. P.J., Jr. 1966. Zoogeography: The geographical distribution of animals. Harvard University, Harvard.

Davis, D.R. 2000. Tineoidea and Gracillarioidea (Lepidoptera). Pp. 469-482. In: J. Llorente Bousquets, E. González and N. Papavero (Eds.). Biodiversidad, taxonomía y biogeografia de artrópodos de México: Hacia una sintesis de su conocimiento, Vol. II. Facultad de Ciencias, UNAM, Mexico, D.F.

Díaz, N.B. \& F.E. Gallardo. 2002. Cynipoidea. Pp. 617- 630. In: J. Llorente Bousquets and J. J. Morrone (Eds.). Biodiversidad, taxonomía y biogeografia de artrópodos de México: Hacia una síntesis de su conocimiento, Vol. III. Facultad de Ciencias, UNAM, Mexico, D.F.

Dirzo, R. 1992. Diversidad florística y estado de conservación de las selvas tropicales de México. Pp. 283-290. In: Sarukhan, J. \& R. Dirzo (Eds.). México ante los retos de la biodiversidad. Conabio, Mexico, D.F.

Dunlop, J.A. 2002. Phylogeny of Chelicerata. Pp. 117-141. In: J. Llorente Bousquets and J. J. Morrone (Eds.). Biodiversidad, taxonomía y biogeografia de artrópodos de México: Hacia una sintesis de su conocimiento, Vol. III. Facultad de Ciencias, UNAM, Mexico, D.F.

Engel, M. 2004. Zoraptera. Pp. 637-640. In: J. Llorente Bousquets, J.J. Morrone, O. YáñezOrdóñez and I. Vargas Fernández (Eds.). Biodiversidad, taxonomía y biogeografía de artrópodos de México: Hacia una síntesis de su conocimiento, Vol. IV. Facultad de Ciencias, UNAM, Mexico, D.F.

Equihua Martínez, A. \& A. Burgos Solorio. 2002. Scolytidae. Pp. 539-557. In: J. Llorente Bousquets and J. J. Morrone (Eds.). Biodiversidad, taxonomía y biogeografía de artrópodos de México: Hacia una síntesis de su conocimiento, Vol. III. Facultad de Ciencias, UNAM, Mexico, D.F.

Espinosa, D., J.J. Morrone, C. Aguilar Zúñiga \& J. Llorente Bousquets. 2000. Regionalización biogeográfica de México: Provincias bióticas. Pp. 61-94. In: J. Llorente Bousquets, E. González and N. Papavero (Eds.). Biodiversidad, taxonomía y biogeografía de artrópodos de México: Hacia una sintesis de su conocimiento, Vol. II. Facultad de Ciencias, UNAM, Mexico, D.F.

Fitzgerald, S.J. 2000. Bibionidae (Diptera). Pp. 627-634. In: J. Llorente Bousquets, E. González and N. Papavero (Eds.). Biodiversidad, taxonomía y biogeografía de artrópodos de México: Hacia una síntesis de su conocimiento, Vol. II. Facultad de Ciencias, UNAM, Mexico, D.F. 
Fursov, V.N., J.M. Coronado Blanco \& E. Ruiz Cancino. 2004. Trichogrammatidae (Hymenoptera). Pp. 747-752. In: J. Llorente Bousquets, J.J. Morrone, O. Yáñez-Ordóñez and I. Vargas Fernández (Eds.). Biodiversidad, taxonomía y biogeografía de artrópodos de México: Hacia una síntesis de su conocimiento, Vol. IV. Facultad de Ciencias, UNAM, Mexico, D.F.

Furth, D.G. 2004. Alticinae (Coleoptera: Chrysomelidae). Pp. 669-684. In: J. Llorente Bousquets, J.J. Morrone, O. Yáñez-Ordóñez and I. Vargas Fernández (Eds.). Biodiversidad, taxonomía y biogeografía de artrópodos de México: Hacia una síntesis de su conocimiento, Vol. IV. Facultad de Ciencias, UNAM, Mexico, D.F.

Godman, F.D. \& O. Salvin (eds.). 1879-1915. Biologia Centrali-Americana. Dulau and Co., London.

González-Hernández, A. 2000. Chalcidoidea (Hymenoptera). Pp. 649-659. In: J. Llorente Bousquets, E. González and N. Papavero (Eds.). Biodiversidad, taxonomía y biogeografia de artrópodos de México: Hacia una sintesis de su conocimiento, Vol. II. Facultad de Ciencias, UNAM, Mexico, D.F.

González Soriano, E. \& R. Novelo Gutiérrez. 1996. Odonata. Pp. 147-167. In: J. Llorente Bousquets, A.N. García Aldrete and E. González Soriano (Eds.). Biodiversidad, taxonomía y biogeografía de artrópodos de México: Hacia una sintesis de su conocimiento. Instituto de Biología, UNAM, Mexico, D.F.

Gutiérrez-Velázquez, A.L., R. Acosta Gutiérrez \& L. Ortiz Lozano. 2006. Patrones de distribución del orden Siphonaptera. Pp. 591-627. In: J.J. Morrone and J. Llorente Bousquets (Eds.). Componentes bióticos principales de la entomofauna mexicana. Vols. I y II. Las Prensas de Ciencias, UNAM, México, D.F.

Halffter, G. 1962. Explicación preliminar de la distribución geográfica de los Scarabaeidae mexicanos. Acta Zoológica Mexicana 5: 1-17.

Halffter, G. 1965. Algunas ideas acerca de la zoogeografía de América. Rev. Soc. Mex. Hist. Nat. 26: 1-16.

Halffter, G. 1972. Eléments anciens de l'entomofaune neotropicale: Ses implications biogéographiques. Pp. 1-40. In: Biogeographie et liasons intercontinentales au cours du Mésozoique. 17me Congr. Int. Zool., Monte Carlo.

Halffter, G. 1974. Eléments anciens de l'entomofaune neotropicale: Ses implications biogéographiques. Quaest. Entomol. 10: 223-262.

Halffter, G. 1976. Distribución de los insectos en la zona de transición mexicana: Relaciones con la entomofauna de Norteamérica. Folia Entomol. Mex. 35: 1-64.

Halffter, G. 1978. Un nuevo patrón de dispersión en la zona de transición mexicana: El mesoamericano de montaña. Folia Entomol. Mex. 39-40: 219-222.

Halffter, G. 1987. Biogeography of the montane entomofauna of Mexico and Central America. Annu. Rev. Entomol. 32: 95-114.

Halffter, G. 2003. Biogeografía de la entomofauna de montaña de México y América Central. Pp. 87-97 in Morrone, J. J. and J. Llorente Bousquets (Eds.). Una perspectiva latinoamericana de la biogeografia. Las Prensas de Ciencias, Facultad de Ciencias, UNAM, Mexico, D.F. 
Hernández-Ortiz, V. 1996. Tephritidae (Diptera). Pp. 603-617. In: J. Llorente Bousquets, A.N. García Aldrete and E. González Soriano (Eds.). Biodiversidad, taxonomía y biogeografía de artrópodos de México: Hacia una síntesis de su conocimiento. Instituto de Biología, UNAM, Mexico, D.F.

Hespenheide, H.A. 1996. Buprestidae (Coleoptera). Pp. 411-421. In: J. Llorente Bousquets, A.N. García Aldrete and E. González Soriano (Eds.). Biodiversidad, taxonomía y biogeografía de artrópodos de México: Hacia una síntesis de su conocimiento. Instituto de Biología, UNAM, Mexico, D.F.

Hoffmann, A. \& M.G. López-Campos. 2002. Acari. Pp. 223-276. In: J. Llorente Bousquets and J. J. Morrone (Eds.). Biodiversidad, taxonomía y biogeografía de artrópodos de México: Hacia una síntesis de su conocimiento, Vol. III. Facultad de Ciencias, UNAM, Mexico, D.F.

Ibáñez-Bernal, S. 2000. Psychodidae (Diptera). Pp. 607-626. In: J. Llorente Bousquets, E. González and N. Papavero (Eds.). Biodiversidad, taxonomía y biogeografía de artrópodos de México: Hacia una síntesis de su conocimiento, Vol. II. Facultad de Ciencias, UNAM, Mexico, D.F.

Ibáñez-Bernal, S. \& S. Coscarón. 1996. Simuliidae (Diptera). Pp. 579-589. In: J. Llorente Bousquets, A.N. García Aldrete and E. González Soriano (Eds.). Biodiversidad, taxonomía y biogeografia de artrópodos de México: Hacia una síntesis de su conocimiento. Instituto de Biología, UNAM, Mexico, D.F.

Ibáñez-Bernal, S. \& S. Coscarón. 2000. Tabanidae (Diptera). Pp. 593-606. In: J. Llorente Bousquets, E. González and N. Papavero (Eds.). Biodiversidad, taxonomía y biogeografía de artrópodos de México: Hacia una síntesis de su conocimiento, Vol. II. Facultad de Ciencias, UNAM, Mexico, D.F.

Ibáñez-Bernal, S., V. Hernández-Ortiz \& L.M. Martín del Campo. 2004. Dolichopodidae (Diptera). Pp. 759-765. In: J. Llorente Bousquets, J.J. Morrone, O. Yáñez-Ordóñez and I. Vargas Fernández (Eds.). Biodiversidad, taxonomía y biogeografía de artrópodos de México: Hacia una sintesis de su conocimiento, Vol. IV. Facultad de Ciencias, UNAM, Mexico, D.F.

Ibáñez-Bernal, S., D. Strickman \& C. Martínez-Campos. 1996a. Culicidae (Diptera). Pp. 591-602. In: J. Llorente Bousquets, A.N. García Aldrete and E. González Soriano (Eds.). Biodiversidad, taxonomía y biogeografía de artrópodos de México: Hacia una sintesis de su conocimiento. Instituto de Biología, UNAM, Mexico, D.F.

Ibáñez-Bernal, S., W.W. Wirth \& H. Huerta-Jiménez. 1996b. Ceratopogonidae (Diptera). Pp. 567-577. In: J. Llorente Bousquets, A.N. García Aldrete and E. González Soriano (Eds.). Biodiversidad, taxonomía y biogeografía de artrópodos de México: Hacia una sintesis de su conocimiento. Instituto de Biología, UNAM, Mexico, D.F.

Jiménez, M.L. 1996. Araneae. Pp. 83-101. In: J. Llorente Bousquets, A.N. García Aldrete and E. González Soriano (Eds.). Biodiversidad, taxonomía y biogeografia de artrópodos de México: Hacia una síntesis de su conocimiento. Instituto de Biología, UNAM, Mexico, D.F.

Johansen, R.M. \& A. Mojica-Guzmán. 1996. Thysanoptera. Pp. 245-273. In: J. Llorente Bousquets, A.N. García Aldrete and E. González Soriano (Eds.). Biodiversidad, 
taxonomía y biogeografía de artrópodos de México: Hacia una sintesis de su conocimiento. Instituto de Biología, UNAM, Mexico, D.F.

Kasparyan, D.R. \& E. Ruiz Cancino. 2004. Adenda a Ichneumonidae (Hymenoptera). Pp. 721-723. In: J. Llorente Bousquets, J.J. Morrone, O. Yáñez-Ordóñez and I. Vargas Fernández (Eds.). Biodiversidad, taxonomía y biogeografia de artrópodos de México: Hacia una sintesis de su conocimiento, Vol. IV. Facultad de Ciencias, UNAM, Mexico, D.F.

Kury, A.B. \& J.C. Cokendolpher. 2000. Opiliones. Pp. 137-157. In: J. Llorente Bousquets, E. González and N. Papavero (Eds.). Biodiversidad, taxonomía y biogeografía de artrópodos de México: Hacia una sintesis de su conocimiento, Vol. II. Facultad de Ciencias, UNAM, Mexico, D.F.

León-Cortés, J.L. 2000. Sphingoidea (Lepidoptera). Pp. 483-500. In: J. Llorente Bousquets, E. González and N. Papavero (Eds.). Biodiversidad, taxonomía y biogeografía de artrópodos de México: Hacia una síntesis de su conocimiento, Vol. II. Facultad de Ciencias, UNAM, Mexico, D.F.

León-Cortés, J.L., I.J. Kitching \& A. Molina-Martínez. 2006. Distributional patterns of the family Sphingidae (Lepidoptera). Pp. 629-647. In: J.J. Morrone and J. Llorente Bousquets (Eds.). Componentes bióticos principales de la entomofauna mexicana. Vols. I y II. Las Prensas de Ciencias, UNAM, Mexico, D.F.

Liebherr, J.K. 1991. A general area cladogram for montane Mexico based on distributions in the Platynine genera Elliptoleus and Calathus (Coleoptera: Carabidae). Proc. Entomol. Soc. Washington 93: 390-406.

Liebherr, J.K. 1994. Biogeographic patterns of montane Mexican and Central American Carabidae (Coleoptera). Can. Entomol. 126: 841-860.

Llorente, J. 1996. Biogeografía de artrópodos de México: ¿Hacia un nuevo enfoque?. Pp. 4156. In: J. Llorente Bousquets, A.N. García Aldrete and E. González Soriano (Eds.). Biodiversidad, taxonomía y biogeografia de artrópodos de México: Hacia una sintesis de su conocimiento. Instituto de Biología, UNAM, Mexico, D.F.

Llorente Bousquets, J., A.N. García Aldrete \& E. González Soriano (Eds.). 1996a. Biodiversidad, taxonomía y biogeografía de artrópodos de México: Hacia una sintesis de su conocimiento. Instituto de Biología, UNAM, Mexico, D.F.

Llorente Bousquets, J., A.N. García Aldrete, E. González Soriano \& C. Cordero. 1996b. Breve panorama de la taxonomía de artrópodos en México. Pp. 3-14. In: J. Llorente Bousquets, A.N. García Aldrete and E. González Soriano (Eds.). Biodiversidad, taxonomía y biogeografía de artrópodos de México: Hacia una síntesis de su conocimiento. Instituto de Biología, UNAM, Mexico, D.F.

Llorente Bousquets, J., A. Luis Martínez, I. Vargas Fernández \& J. Soberón Mainero. 1996c. Papilionoidea (Lepidoptera). Pp. 531-548. In: J. Llorente Bousquets, A.N. García Aldrete and E. González Soriano (Eds.). Biodiversidad, taxonomía y biogeografía de artrópodos de México: Hacia una síntesis de su conocimiento. Instituto de Biología, UNAM, Mexico, D.F.

Llorente Bousquets, J., E. González \& N. Papavero (Eds.). 2000. Biodiversidad, taxonomía y biogeografía de artrópodos de México: Hacia una síntesis de su conocimiento, Vol. II. Facultad de Ciencias, UNAM, Mexico, D.F. 
Llorente Bousquets, J. \& J.J. Morrone (Eds.). 2002. Biodiversidad, taxonomía y biogeografía de artrópodos de México: Hacia una sintesis de su conocimiento, Vol. III. Facultad de Ciencias, UNAM, Mexico, D.F.

Llorente Bousquets, J., J.J. Morrone, O. Yáñez-Ordóñez \& I. Vargas Fernández (Eds.). 2004. Biodiversidad, taxonomía y biogeografía de artrópodos de México: Hacia una sintesis de su conocimiento, Vol. IV. Facultad de Ciencias, UNAM, Mexico, D.F.

Llorente-Bousquets, J., M. Trujano-Ortega, A. Luis-Martínez, J. Castro \& I. VargasFernández. 2006. Patrones de distribución de la familia Pieridae (Lepidoptera). Pp. 715770. In: J.J. Morrone and J. Llorente Bousquets (Eds.). Componentes bióticos principales de la entomofauna mexicana. Vols. I y II. Las Prensas de Ciencias, UNAM, Mexico, D.F.

Loiácono, M.S. \& C.B. Margaría. 2002. Proctotrupoidea. Pp. 597-616. In: J. Llorente Bousquets and J. J. Morrone (Eds.). Biodiversidad, taxonomía y biogeografía de artrópodos de México: Hacia una síntesis de su conocimiento, Vol. III. Facultad de Ciencias, UNAM, Mexico, D.F.

Lourenço, W.R. \& W.D. Sissom. 2000. Scorpiones. Pp. 115-135. In: J. Llorente Bousquets, E. González and N. Papavero (Eds.). Biodiversidad, taxonomía y biogeografía de artrópodos de México: Hacia una síntesis de su conocimiento, Vol. II. Facultad de Ciencias, UNAM, Mexico, D.F.

Luis, A.M., I. Vargas \& J. Llorente. 1991. Lepidopterofauna de Oaxaca I: Distribución y fenología de Papilionoidea de la Sierra de Juárez. Pub. Esp. Mus. Zool., UNAM 3: 1-118.

Luis-Martínez, A., M. Trujano, J. Llorente-Bousquets \& I. Vargas-Fernández. 2006. Patrones de distribución de las subfamilias Danainae, Apaturinae, Biblidinae y Heliconiinae (Lepidoptera: Nymphalidaee). Pp. 771-865. In: J.J. Morrone and J. Llorente Bousquets (Eds.). Componentes bióticos principales de la entomofauna mexicana. Vols. I y II. Las Prensas de Ciencias, UNAM, Mexico, D.F.

Luna, I., O. Alcántara, J.J. Morrone \& D. Espinosa. 2000. Track analysis and conservation priorities in the cloud forests of Hidalgo, Mexico. Divers. Distrib. 6: 137-143.

Luna, I. \& J. Llorente (Eds.). 1993. Historia natural del Parque Ecológico Estatal Omiltemi, Chilpancingo, Guerrero, México. UNAM, Mexico, D.F.

Márquez, J. \& J. Asiain. 2006. Patrones de distribución de la familia Staphylinidae (Coleoptera). Pp. 157-236. In: J.J. Morrone and J. Llorente Bousquets (Eds.). Componentes bióticos principales de la entomofauna mexicana. Vols. I y II. Las Prensas de Ciencias, UNAM, Mexico, D.F.

Marshall, C.J. \& J.K. Liebherr. 2000. Cladistic biogeography of the Mexican transition zone. J. Biogeogr. 27: 203-216.

McCafferty, W.P. \& C.R. Lugo-Ortiz. 1996. Ephemeroptera. Pp. 133-145. In: J. Llorente Bousquets, A.N. García Aldrete and E. González Soriano (Eds.). Biodiversidad, taxonomía y biogeografía de artrópodos de México: Hacia una síntesis de su conocimiento. Instituto de Biología, UNAM, Mexico, D.F.

Miller, J.Y. 2000. Castniidae (Lepidoptera). Pp. 527-533. In: J. Llorente Bousquets, E. González and N. Papavero (Eds.). Biodiversidad, taxonomía y biogeografía de artrópodos de México: Hacia una síntesis de su conocimiento, Vol. II. Facultad de Ciencias, UNAM, Mexico, D.F. 
Mittermeier, R.A. 1988. Primate diversity and the tropical forest: Case studies from Brazil and Madagascar and the importance of the megadiversity countries. Pp. 145-154. In: Wilson, E. (Ed.). Biodiversity. National Academic Press, Washington, D.C.

Mockford, E.L. \& A. García Aldrete. 1996. Psocoptera. Pp. 175-205. In: J. Llorente Bousquets, A.N. García Aldrete and E. González Soriano (Eds.). Biodiversidad, taxonomía y biogeografia de artrópodos de México: Hacia una sintesis de su conocimiento. Instituto de Biología, UNAM, Mexico, D.F.

Morón, M.A. 1996a. Melolonthidae (Coleoptera). Pp. 287-307. In: J. Llorente Bousquets, A.N. García Aldrete and E. González Soriano (Eds.). Biodiversidad, taxonomía y biogeografía de artrópodos de México: Hacia una síntesis de su conocimiento. Instituto de Biología, UNAM, Mexico, D.F.

Morón, M.A. 1996b. Scarabaeidae (Coleoptera). Pp. 309-328. In: J. Llorente Bousquets, A.N. García Aldrete and E. González Soriano (Eds.). Biodiversidad, taxonomía y biogeografía de artrópodos de México: Hacia una síntesis de su conocimiento. Instituto de Biología, UNAM, Mexico, D.F.

Morón, M.A. 2006a. Patrones de distribución de la familia Scarabaeidae (Coleoptera). Pp. 271-293. In: J.J. Morrone and J. Llorente Bousquets (Eds.). Componentes bióticos principales de la entomofauna mexicana. Vols. I y II. Las Prensas de Ciencias, UNAM, México, D.F.

Morón, M.A. 2006b. Patrones de distribución de la familia Melolonthidae (Coleoptera). Pp. 295-331. In: J.J. Morrone and J. Llorente Bousquets (Eds.). Componentes bióticos principales de la entomofauna mexicana. Vols. I y II. Las Prensas de Ciencias, UNAM, Mexico, D.F.

Morrone, J.J. 1999. How can biogeography and cladistics interact for the selection of areas for biodiversity conservation?: A view from Andean weevils (Coleoptera: Curculionidae). Biogeographica 75: 89-96.

Morrone, J.J. 2001a. Biogeografía de América Latina y el Caribe. Manuales y Tesis SEA, nro. 3, Zaragoza (Spain).

Morrone, J.J. 2001b. Homology, biogeography and areas of endemism. Divers. Distrib. 7: 297-300.

Morrone, J.J. 2002. Nemonychidae. Pp. 535-538. In: J. Llorente Bousquets and J. J. Morrone (Eds.). Biodiversidad, taxonomía y biogeografia de artrópodos de México: Hacia una sintesis de su conocimiento, Vol. III. Facultad de Ciencias, UNAM, Mexico, D.F.

Morrone, J.J. 2004a. Belidae (Coleoptera). Pp. 685-689. In: J. Llorente Bousquets, J.J. Morrone, O. Yáñez-Ordóñez and I. Vargas Fernández (Eds.). Biodiversidad, taxonomía y biogeografía de artrópodos de México: Hacia una síntesis de su conocimiento, Vol. IV. Facultad de Ciencias, UNAM, Mexico, D.F.

Morrone, J.J. 2004b. Erirhinidae (Coleoptera). Pp. 701-704. In: J. Llorente Bousquets, J.J. Morrone, O. Yáñez-Ordóñez and I. Vargas Fernández (Eds.). Biodiversidad, taxonomía y biogeografía de artrópodos de México: Hacia una síntesis de su conocimiento, Vol. IV. Facultad de Ciencias, UNAM, Mexico, D.F.

Morrone, J.J. 2004c. Homología biogeográfica: Las coordenadas espaciales de la vida. Cuadernos del Instituto de Biología 37, Instituto de Biología, UNAM, Mexico, D.F. 
Morrone, J.J. 2005. Hacia una síntesis biogeográfica de México. Rev. Mex. Biodivers. 76: 207-252.

Morrone, J.J. 2006. Biogeographic areas and transition zones of Latin America and the Caribbean Islands, based on panbiogeographic and cladistic analyses of the entomofauna. Annu. Rev. Entomol. 51: 467-494.

Morrone, J.J. \& P.I. Cuevas. 2004. Dryophthoridae (Coleoptera). Pp. 705-712. In: J. Llorente Bousquets, J.J. Morrone, O. Yáñez-Ordóñez and I. Vargas Fernández (Eds.). Biodiversidad, taxonomía y biogeografía de artrópodos de México: Hacia una síntesis de su conocimiento, Vol. IV. Facultad de Ciencias, UNAM, Mexico, D.F.

Morrone, J.J. \& D. Espinosa. 1998. La relevancia de los atlas biogeográficos para la conservación de la biodiversidad mexicana. Ciencia (México) 49: 12-16.

Morrone, J.J., D. Espinosa, C. Aguilar \& J. Llorente Bousquets. 1999. Preliminary classification of the Mexican biogeographic provinces: A parsimony analysis of endemicity based on plant, insect, and bird taxa. Southwest. Nat. 44: 507-514.

Morrone, J.J, D. Espinosa \& J. Llorente Bousquets. 2002. Mexican biogeographic provinces: Preliminary scheme, general characterizations, and synonymies. Acta Zool. Mex. (n.s.) 85: 83-108.

Morrone, J.J. \& J. Llorente Bousquets (Eds.). 2006. Componentes bióticos principales de la entomofauna mexicana. Vols. I y II. Las Prensas de Ciencias, UNAM, Mexico, D.F.

Morrone, J.J. \& J. Llorente Bousquets. 2006. Conclusiones. Pp. 1011-1025. In: J.J. Morrone and J. Llorente Bousquets (Eds.). Componentes bióticos principales de la entomofauna mexicana. Vols. I y II. Las Prensas de Ciencias, UNAM, Mexico, D.F.

Morrone, J.J. \& J. Márquez. 2001. Halffter's Mexican Transition Zone, beetle generalized tracks, and geographical homology. J. Biogeogr. 28: 635-650.

Morrone, J.J. \& J. Márquez. 2003. Aproximación a un Atlas Biogeográfico Mexicano: Componentes bióticos principales y provincias biogeográficas. Pp. 217-220. In: J.J. Morrone and J. Llorente Bousquets (Eds.). Una perspectiva latinoamericana de la biogeografía. Las Prensas de Ciencias, Facultad de Ciencias, UNAM, Mexico, D.F.

Myartseva, S.N., E. Ruiz Cancino \& J.M. Coronado Blanco. 2004. Aphelinidae (Hymenoptera). Pp. 753-757. In: J. Llorente Bousquets, J.J. Morrone, O. Yáñez-Ordóñez and I. Vargas Fernández (Eds.). Biodiversidad, taxonomía y biogeografia de artrópodos de México: Hacia una síntesis de su conocimiento, Vol. IV. Facultad de Ciencias, UNAM, Mexico, D.F.

Navarrete-Heredia, J.L. \& R.G. Beutel. 2006. Patrones de distribución de la familia Lepiceridae (Coleoptera). Pp. 89-92. In: J.J. Morrone and J. Llorente Bousquets (Eds.). Componentes bióticos principales de la entomofauna mexicana. Vols. I y II. Las Prensas de Ciencias, UNAM, Mexico, D.F.

Navarrete-Heredia, J.L. \& A. Burgos-Solorio. 2000. Ciidae (Coleoptera). Pp. 413-420. In: J. Llorente Bousquets, E. González and N. Papavero (Eds.). Biodiversidad, taxonomía y biogeografía de artrópodos de México: Hacia una síntesis de su conocimiento, Vol. II. Facultad de Ciencias, UNAM, Mexico, D.F.

Navarrete-Heredia, J.L. \& H.E. Fierros-López. 2000. Silphidae (Coleoptera). Pp. 401-412. In: J. Llorente Bousquets, E. González and N. Papavero (Eds.). Biodiversidad, taxonomía 
Morrone \& Márquez: Biodiversity of mexican terrestrial arthropods

y biogeografía de artrópodos de México: Hacia una sintesis de su conocimiento, Vol. II. Facultad de Ciencias, UNAM, Mexico, D.F.

Navarrete-Heredia, J.L. \& A.F. Newton, Jr. 1996. Staphylinidae (Coleoptera). Pp. 369-380. In: J. Llorente Bousquets, A.N. García Aldrete and E. González Soriano (Eds.). Biodiversidad, taxonomía y biogeografía de artrópodos de México: Hacia una síntesis de su conocimiento. Instituto de Biología, UNAM, Mexico, D.F.

Navarrete-Heredia, J.L. \& A.F. Newton, Jr. 2002. Agyrtidae. Pp. 493-498. In: J. Llorente Bousquets and J. J. Morrone (Eds.). Biodiversidad, taxonomía y biogeografia de artrópodos de México: Hacia una síntesis de su conocimiento, Vol. III. Facultad de Ciencias, UNAM, Mexico, D.F.

Navarrete-Heredia, J.L. \& G.A. Quiroz-Rocha. 2004. Hydraenidae (Coleoptera). Pp. 641647. In: J. Llorente Bousquets, J.J. Morrone, O. Yáñez-Ordóñez and I. Vargas Fernández (Eds.). Biodiversidad, taxonomía y biogeografia de artrópodos de México: Hacia una sintesis de su conocimiento, Vol. IV. Facultad de Ciencias, UNAM, Mexico, D.F.

Navarrete-Heredia, J.L., G.A. Quiroz-Rocha. P.W. Kovarik, M.S. Cayerino, A. Tischechkin \& M. Vásquez-Bolaños. 2004a. Histeridae (Coleoptera). Pp. 649-658. In: J. Llorente Bousquets, J.J. Morrone, O. Yáñez-Ordóñez and I. Vargas Fernández (Eds.). Biodiversidad, taxonomía y biogeografía de artrópodos de México: Hacia una síntesis de su conocimiento, Vol. IV. Facultad de Ciencias, UNAM, Mexico, D.F.

Navarrete-Heredia, J.L., P.E. Skelley \& G.A. Quiroz-Rocha. 2004b. Erotylidae (Coleoptera). Pp. 659-667. In: J. Llorente Bousquets, J.J. Morrone, O. Yáñez-Ordóñez and I. Vargas Fernández (Eds.). Biodiversidad, taxonomía y biogeografía de artrópodos de México: Hacia una síntesis de su conocimiento, Vol. IV. Facultad de Ciencias, UNAM, Mexico, D.F.

Noguera, F.A. \& J.A. Chemsack. 1996. Cerambycidae (Coleoptera). Pp. 381-409. In: J. Llorente Bousquets, A.N. García Aldrete and E. González Soriano (Eds.). Biodiversidad, taxonomía y biogeografía de artrópodos de México: Hacia una sintesis de su conocimiento. Instituto de Biología, UNAM, Mexico, D.F.

O’Brien, L., D.R. Miller, T.E. Moore, R. Peña Martínez, M.M. Yang \& D.R. Miller. 1996. Homoptera. Pp. 208-244. In: J. Llorente Bousquets, A.N. García Aldrete and E. González Soriano (Eds.). Biodiversidad, taxonomía y biogeografia de artrópodos de México: Hacia una síntesis de su conocimiento. Instituto de Biología, UNAM, Mexico, D.F.

Oñate-Ocaña, L., M. Trujano-Ortega, J. Llorente-Bousquets, A. Luis-Martínez \& I. Vargas-Fernández. 2006. Patrones de distribución de la familia Papilionidae (Lepidoptera). Pp. 661-714. In: J.J. Morrone and J. Llorente Bousquets (Eds.). Componentes bióticos principales de la entomofauna mexicana. Vols. I y II. Las Prensas de Ciencias, UNAM, Mexico, D.F.

Ordóñez Reséndiz, M.M. 2006. Patrones de distribución de la familia Carabidae (Coleoptera). Pp. 93-155. In: J.J. Morrone and J. Llorente Bousquets (Eds.). Componentes bióticos principales de la entomofauna mexicana. Vols. I y II. Las Prensas de Ciencias, UNAM, Mexico, D.F.

Ordóñez Reséndiz, M.M. \& M.A. Eligio García. 2006. Patrones de distribución de la familia Chrysomelidae (Coleoptera). Pp. 475-514. In: J.J. Morrone and J. Llorente 
Bousquets (Eds.). Componentes bióticos principales de la entomofauna mexicana. Vols. I y II. Las Prensas de Ciencias, UNAM, Mexico, D.F.

Oswald, J.D., A. Contreras-Ramos \& N.D. Penny. 2002. Neuroptera (Neuropterida). Pp. 559-581. In: J. Llorente Bousquets and J. J. Morrone (Eds.). Biodiversidad, taxonomía y biogeografía de artrópodos de México: Hacia una síntesis de su conocimiento, Vol. III. Facultad de Ciencias, UNAM, Mexico, D.F.

Palacios-Vargas, J.G. 2000a. Protura y Diplura. Pp. 275-281. In: J. Llorente Bousquets, E. González and N. Papavero (Eds.). Biodiversidad, taxonomía y biogeografía de artrópodos de México: Hacia una síntesis de su conocimiento, Vol. II. Facultad de Ciencias, UNAM, Mexico, D.F.

Palacios-Vargas, J.G. 2000b. Archaeognatha y Zygentoma. Pp. 285-291. In: J. Llorente Bousquets, E. González and N. Papavero (Eds.). Biodiversidad, taxonomía y biogeografía de artrópodos de México: Hacia una síntesis de su conocimiento, Vol. II. Facultad de Ciencias, UNAM, Mexico, D.F.

Palacios-Vargas, J.G., G. Castaño-Meneses \& B.E. Mejía-Recamier. 2000. Collembola. Pp. 249-273. In: J. Llorente Bousquets, E. González and N. Papavero (Eds.). Biodiversidad, taxonomía y biogeografía de artrópodos de México: Hacia una sintesis de su conocimiento, Vol. II. Facultad de Ciencias, UNAM, Mexico, D.F.

Palacios-Vargas, J.G. \& R. Iglesias. 2004. Oribatei (Acari). Pp. 431-468. In: J. Llorente Bousquets, J.J. Morrone, O. Yáñez-Ordóñez and I. Va rgas Fernández (Eds.). Biodiversidad, taxonomía y biogeografía de artrópodos de México: Hacia una síntesis de su conocimiento, Vol. IV. Facultad de Ciencias, UNAM, Mexico, D.F.

Papavero, N. 1996. Mydidae (Diptera). Pp. 619-634. In: J. Llorente Bousquets, A.N. García Aldrete and E. González Soriano (Eds.). Biodiversidad, taxonomía y biogeografía de artrópodos de México: Hacia una síntesis de su conocimiento. Instituto de Biología, UNAM, Mexico, D.F.

Peck, S.B. 2000. Leiodidae (Coleoptera). Pp. 439-452. In: J. Llorente Bousquets, E. González and N. Papavero (Eds.). Biodiversidad, taxonomía y biogeografía de artrópodos de México: Hacia una síntesis de su conocimiento, Vol. II. Facultad de Ciencias, UNAM, Mexico, D.F.

Ponce Ulloa, H. \& J.E. Llorente Bousquets. 1996. Siphonaptera. Pp. 553-565. In: J. Llorente Bousquets, A.N. García Aldrete and E. González Soriano (Eds.). Biodiversidad, taxonomía y biogeografía de artrópodos de México: Hacia una síntesis de su conocimiento. Instituto de Biología, UNAM, Mexico, D.F.

Razowski, J. 1996. Tortricidae (Lepidoptera). Pp. 513-520. In: J. Llorente Bousquets, A.N. García Aldrete and E. González Soriano (Eds.). Biodiversidad, taxonomía y biogeografía de artrópodos de México: Hacia una sintesis de su conocimiento. Instituto de Biología, UNAM, Mexico, D.F.

Reyes-Castillo, P. 2002a. Passalidae. Pp. 467-483. In: J. Llorente Bousquets and J. J. Morrone (Eds.). Biodiversidad, taxonomía y biogeografía de artrópodos de México: Hacia una sintesis de su conocimiento, Vol. III. Facultad de Ciencias, UNAM, Mexico, D.F.

Reyes-Castillo, P. 2002b. Lucanidae. Pp. 485-492. In: J. Llorente Bousquets and J. J. Morrone (Eds.). Biodiversidad, taxonomía y biogeografía de artrópodos de México: 
Morrone \& Márquez: Biodiversity of mexican terrestrial arthropods

Hacia una síntesis de su conocimiento, Vol. III. Facultad de Ciencias, UNAM, Mexico, D.F.

Reyes-Castillo, P. 2003. Las ideas biogeográficas de Gonzalo Halffter: Importancia e impacto. Pp. 99-108. In: Morrone, J. J. \& J. Llorente Bousquets (Eds.). Una perspectiva latinoamericana de la biogeografía. Las Prensas de Ciencias, Facultad de Ciencias, UNAM, Mexico, D.F.

Reyes-Castillo, P., C.V. Rojas-Gómez \& H. Vázquez. 2006. Patrones de distribución de la familia Passalidae (Coleoptera). Pp. 237-270. In: J.J. Morrone and J. Llorente Bousquets (Eds.). Componentes bióticos principales de la entomofauna mexicana. Vols. I y II. Las Prensas de Ciencias, UNAM, Mexico, D.F.

Rodríguez Palafox, A. 1996. Vespidae (Hymenoptera). Pp. 465-482. In: J. Llorente Bousquets, A.N. García Aldrete and E. González Soriano (Eds.). Biodiversidad, taxonomía y biogeografía de artrópodos de México: Hacia una síntesis de su conocimiento. Instituto de Biología, UNAM, Mexico, D.F.

Rojas Fernández P. 1996. Formicidae (Hymenoptera). Pp. 483-500. In: J. Llorente Bousquets, A.N. García Aldrete and E. González Soriano (Eds.). Biodiversidad, taxonomía y biogeografía de artrópodos de México: Hacia una síntesis de su conocimiento. Instituto de Biología, UNAM, Mexico, D.F.

Romero Nápoles, J. 2002. Bruchidae. Pp. 513-534. In: J. Llorente Bousquets and J. J. Morrone (Eds.). Biodiversidad, taxonomía y biogeografía de artrópodos de México: Hacia una síntesis de su conocimiento, Vol. III. Facultad de Ciencias, UNAM, Mexico, D.F.

Ruiz Cancino, E. \& J.M. Coronado Blanco. 2002. Pompiloidea. Pp. 647-656. In: J. Llorente Bousquets and J. J. Morrone (Eds.). Biodiversidad, taxonomía y biogeografía de artrópodos de México: Hacia una síntesis de su conocimiento, Vol. III. Facultad de Ciencias, UNAM, Mexico, D.F.

Ruiz Cancino, E., J.M. Coronado Blanco, S.N. Myartseva \& J.F. Lunas Salas. 2004. Adenda a Chalcidoidea (Hymenoptera). Pp. 725-734. In: J. Llorente Bousquets, J.J. Morrone, O. Yáñez-Ordóñez and I. Vargas Fernández (Eds.). Biodiversidad, taxonomía y biogeografía de artrópodos de México: Hacia una síntesis de su conocimiento, Vol. IV. Facultad de Ciencias, UNAM, Mexico, D.F.

Ruiz Cancino, E., J.M. Coronado Blanco, S.E. Varela Fuentes \& J.V. Horta Vega. 2002 a. Sphecoidea. Pp. 656-670. In: J. Llorente Bousquets and J. J. Morrone (Eds.). Biodiversidad, taxonomía y biogeografía de artrópodos de México: Hacia una sintesis de su conocimiento, Vol. III. Facultad de Ciencias, UNAM, Mexico, D.F.

Ruiz Cancino, E., D.R. Kasparyan \& J.M. Coronado Blanco. 2002b. Ichneumonidae. Pp. 631-646. In: J. Llorente Bousquets and J. J. Morrone (Eds.). Biodiversidad, taxonomía y biogeografía de artrópodos de México: Hacia una síntesis de su conocimiento, Vol. III. Facultad de Ciencias, UNAM, Mexico, D.F.

Sakai, S. 2004. Dermaptera. Pp. 627-636. In: J. Llorente Bousquets, J.J. Morrone, O. YáñezOrdóñez and I. Vargas Fernández (Eds.). Biodiversidad, taxonomía y biogeografía de artrópodos de México: Hacia una síntesis de su conocimiento, Vol. IV. Facultad de Ciencias, UNAM, Mexico, D.F. 
Santiago-Fragoso, S. \& P.J. Spangler. 2000. Elmidae (Coleoptera). Pp. 421-438. In: J. Llorente Bousquets, E. González and N. Papavero (Eds.). Biodiversidad, taxonomía y biogeografía de artrópodos de México: Hacia una sintesis de su conocimiento, Vol. II. Facultad de Ciencias, UNAM, Mexico, D.F.

Sclater, P.L. 1858. On the general geographic distribution of the members of the class Aves. J. Linn. Soc., Zool. 2: 130-145.

Simpson, G.G. 1980. Splendid isolation: The curious history of South American mammals. Yale University Press, New Haven and London.

Slater, J.A. \& H. Brailovsky. 2000. Lygaeidae (Hemiptera). Pp. 319-333. In: J. Llorente Bousquets, E. González and N. Papavero (Eds.). Biodiversidad, taxonomía y biogeografia de artrópodos de México: Hacia una sintesis de su conocimiento, Vol. II. Facultad de Ciencias, UNAM, Mexico, D.F.

Solís, M.A. 1996. Pyraloidea (Lepidoptera). Pp. 521-530. In: J. Llorente Bousquets, A.N. García Aldrete and E. González Soriano (Eds.). Biodiversidad, taxonomía y biogeografía de artrópodos de México: Hacia una síntesis de su conocimiento. Instituto de Biología, UNAM, Mexico, D.F.

Szumik, C. 2002. Embioptera. Pp. 441-448. In: J. Llorente Bousquets and J. J. Morrone (Eds.). Biodiversidad, taxonomía y biogeografía de artrópodos de México: Hacia una sintesis de su conocimiento, Vol. III. Facultad de Ciencias, UNAM, Mexico, D.F.

Thomas, D.B. 2000. Pentatomidae (Hemiptera). Pp. 335-352. In: J. Llorente Bousquets, E. González and N. Papavero (Eds.). Biodiversidad, taxonomía y biogeografia de artrópodos de México: Hacia una sintesis de su conocimiento, Vol. II. Facultad de Ciencias, UNAM, Mexico, D.F.

Toledo, V.H. \& A.M. Corona. 2006. Patrones de distribución de la familia Cerambycidae (Coleoptera). Pp. 425-474. In: J.J. Morrone and J. Llorente Bousquets (Eds.). Componentes bióticos principales de la entomofauna mexicana. Vols. I y II. Las Prensas de Ciencias, UNAM, Mexico, D.F.

Toledo, V.M. 1981. Pleistocene changes of vegetation in Mexico. Pp. 93-111. In: Prance, G. T. (Ed.). Biological diversification in the tropics. Columbia University Press, New York.

Triapitsyn, S.V., J.M. Coronado Blanco \& E. Ruiz-Cancino. 2004a. Mymaridae (Hymenoptera). Pp. 743-746. In: J. Llorente Bousquets, J.J. Morrone, O. Yáñez-Ordóñez and I. Vargas Fernández (Eds.). Biodiversidad, taxonomía y biogeografía de artrópodos de México: Hacia una síntesis de su conocimiento, Vol. IV. Facultad de Ciencias, UNAM, Mexico, D.F.

Triapitsyn, S.V., J.M. Coronado Blanco \& E. Ruiz-Cancino. 2004b. Encyrtidae (Hymenoptera). Pp. 735-742. In: J. Llorente Bousquets, J.J. Morrone, O. Yáñez-Ordóñez and I. Vargas Fernández (Eds.). Biodiversidad, taxonomía y biogeografía de artrópodos de México: Hacia una sintesis de su conocimiento, Vol. IV. Facultad de Ciencias, UNAM, Mexico, D.F.

Vargas-Fernández, I., M. Trujano, J. Llorente-Bousquets \& A. Luis-Martínez. 2006. Patrones de distribución de las subfamilias Ithomiinae, Morphinae y Charaxinae (Lepidoptera: Nymphalidae). Pp. 867-943. In: J.J. Morrone and J. Llorente Bousquets (Eds.). Componentes bióticos principales de la entomofauna mexicana. Vols. I y II. Las Prensas de Ciencias, UNAM, Mexico, D.F. 
Vázquez Rojas, I. 1996a. Solifugae. Pp. 75-78. In: J. Llorente Bousquets, A.N. García Aldrete and E. González Soriano (Eds.). Biodiversidad, taxonomía y biogeografía de artrópodos de México: Hacia una síntesis de su conocimiento. Instituto de Biología, UNAM, Mexico, D.F.

Vázquez Rojas, I. 1996b. Ricinulei. Pp. 79-82. In: J. Llorente Bousquets, A.N. García Aldrete and E. González Soriano (Eds.). Biodiversidad, taxonomía y biogeografía de artrópodos de México: Hacia una síntesis de su conocimiento. Instituto de Biología, UNAM, Mexico, D.F.

Vázquez Rojas, I. 1996c. Palpigradi. Pp. 59-61. In: J. Llorente Bousquets, A.N. García Aldrete and E. González Soriano (Eds.). Biodiversidad, taxonomía y biogeografía de artrópodos de México: Hacia una síntesis de su conocimiento. Instituto de Biología, UNAM, Mexico, D.F.

Vázquez Rojas, I. 1996d. Amplypygi. Pp. 71-73. In: J. Llorente Bousquets, A.N. García Aldrete and E. González Soriano (Eds.). Biodiversidad, taxonomía y biogeografía de artrópodos de México: Hacia una síntesis de su conocimiento. Instituto de Biología, UNAM, Mexico, D.F.

Vázquez Rojas, I. 1996e. Uropygi. Pp. 67-69. In: J. Llorente Bousquets, A.N. García Aldrete and E. González Soriano (Eds.). Biodiversidad, taxonomía y biogeografía de artrópodos de México: Hacia una síntesis de su conocimiento. Instituto de Biología, UNAM, Mexico, D.F.

Vázquez Rojas, I. 1996f. Schizomida. Pp. 63-66. In: J. Llorente Bousquets, A.N. García Aldrete and E. González Soriano (Eds.). Biodiversidad, taxonomía y biogeografía de artrópodos de México: Hacia una síntesis de su conocimiento. Instituto de Biología, UNAM, Mexico, D.F.

Wallace, A.R. 1876. The geographical distribution of animals. London.

Warren, A.D. 2000. Hesperioidea (Lepidoptera). Pp. 535-580. In: J. Llorente Bousquets, E. González and N. Papavero (Eds.). Biodiversidad, taxonomía y biogeografía de artrópodos de México: Hacia una síntesis de su conocimiento, Vol. II. Facultad de Ciencias, UNAM, Mexico, D.F.

Wharton, R.A. \& I. Mercado. 2000. Braconidae (Hymenoptera). Pp. 635-647. In: J. Llorente Bousquets, E. González and N. Papavero (Eds.). Biodiversidad, taxonomía y biogeografía de artrópodos de México: Hacia una sintesis de su conocimiento, Vol. II. Facultad de Ciencias, UNAM, Mexico, D.F.

Yánez-Ordóñez, O. \& M. Trujado Ortega. 2006. Patrones de distribución de la tribu Meliponini (Hymenoptera: Apidae). Pp. 563-590. In: J.J. Morrone and J. Llorente Bousquets (Eds.). Componentes bióticos principales de la entomofauna mexicana. Vols. I y II. Las Prensas de Ciencias, UNAM, Mexico, D.F.

Zaragoza Caballero, S. \& A. Mendoza Ramírez. 1996. Malacodermata (Coleoptera). Pp. 353-368. In: J. Llorente Bousquets, A.N. García Aldrete and E. González Soriano (Eds.). Biodiversidad, taxonomía y biogeografía de artrópodos de México: Hacia una sintesis de su conocimiento. Instituto de Biología, UNAM, Mexico, D.F. 
Zaragoza Caballero, S., M.A. Sarmiento Cordero, M.I. Pacheco Rueda \& M.L. Zurita García. 2006. Patrones de distribución de la familia Lycidae (Coleoptera). Pp. 393-424. In: J.J. Morrone and J. Llorente Bousquets (Eds.). Componentes bióticos principales de la entomofauna mexicana. Vols. I y II. Las Prensas de Ciencias, UNAM, Mexico, D.F.

Recibido: 3 de noviembre de 2006

Aceptado: 25 de octubre de 2007 
